Mens

Revue d'histoire intellectuelle et culturelle

\title{
Mathieu Noël. Lionel Groulx et le réseau indépendantiste des années 1930, Montréal, VLB éditeur, 2011, 143 p.
}

\section{Frédéric Boily}

Volume 13, numéro 1, automne 2012

S’approprier le passé des autres : les usages de l'histoire internationale au Québec avant la Révolution tranquille

URI : https://id.erudit.org/iderudit/1019703ar

DOI : https://doi.org/10.7202/1019703ar

Aller au sommaire du numéro

Éditeur(s)

Centre de recherche en civilisation canadienne-française

ISSN

1492-8647 (imprimé)

1927-9299 (numérique)

Découvrir la revue

Citer ce compte rendu

Boily, F. (2012). Compte rendu de [Mathieu Noël. Lionel Groulx et le réseau indépendantiste des années 1930, Montréal, VLB éditeur, 2011, 143 p.] Mens, 13(1), 131-135. https://doi.org/10.7202/1019703ar d'utilisation que vous pouvez consulter en ligne.

https://apropos.erudit.org/fr/usagers/politique-dutilisation/ 


\section{Comptes rendus}

\section{Mathieu Noël. Lionel Groulx et le réseau indépendantiste des années 1930, Montréal, VLB éditeur, 2011, 143 p.}

Depuis une décennie maintenant, le nombre de travaux consacrés à l'œuvre et à la place de Lionel Groulx dans l'histoire de la pensée québécoise et dans le développement du nationalisme ne cesse de grandir. Le plus récent texte est celui d'un jeune historien, Mathieu Noël, qui nous livre ici les résultats de sa recherche effectuée dans le cadre de sa maîtrise, un ouvrage qui vient s'ajouter de belle manière au corpus d'études groulxistes existant. L'objectif poursuivi par Noël consiste à éclairer l'émergence de ce qu'il appelle un réseau indépendantiste, tout en montrant comment les jeunes intellectuels qui le formaient gravitaient autour de Groulx. Ainsi, Noël ne propose pas une nouvelle exégèse de la pensée de Groulx, car il laisse la parole aux jeunes intellectuels qui étaient tournés vers l'indépendance et pour qui la pensée groulxiste apparaissait comme la référence majeure. Au-delà de l'approche ancrée dans la théorie des réseaux, la force de l'ouvrage repose donc sur le dépouillement de la correspondance que les différents membres des groupes étudiés ont entretenue, entre eux et avec leurs aînés.

Dans le premier chapitre, la place qu'a occupée Groulx dans l'émergence de l'indépendantisme sert de fil conducteur à Noël pour effectuer le tour d'horizon de la production intellectuelle des études groulxistes. S'il y a une question qui a tourmenté les historiens et les spécialistes de Groulx, c'est bien celle de comprendre le rôle complexe joué par ce dernier dans la genèse d'un nationalisme ouvertement indépendantiste. Comme l'auteur le rappelle correctement, « la majorité des historiens s'entendent pour affirmer que Groulx s'intéresse au discours indépendantiste, mais que certains aspects de son nationalisme canadien-français l'empêchent d'avaliser cette idéologie » (p. 15). 
C'est pourquoi si les historiens ont reconnu l'importance de ce dernier dans les débats menant au discours indépendantiste, ils ont cependant eu tendance à en minimiser l'importance. De manière générale, ils ont conclu que Groulx restait trop attaché au Canada français, ou bien que sa conception de la nation incluait l'ensemble des francophones et des catholiques disséminés sur tout le territoire canadien (et des États-Unis), ou bien que sa pensée était apolitique, donc imperméable à l'idée de l'indépendance. Or Noël réintègre la variable groulxiste dans l'équation générale, permettant ainsi de comprendre comment s'est développée l'idée d'indépendance au sein des réseaux des années 1930.

Noël suppose que Groulx s'était prononcé sur la question de l'indépendance du Canada français, ce qui l'amène à rejeter l'approche de Gérard Bouchard selon laquelle il existait deux chanoines, l'un disant une chose, l'autre le contredisant presque au même moment. À ses yeux, Bouchard a omis de prendre en compte les contextes d'énonciation des prises de position de Groulx et, surtout, négligé d'effectuer le travail de contextualisation historique. Dans le même ordre d'idées, il écarte l'approche d'André J. Bélanger sur l'apolitisme des idéologies, tout comme il nuance les positions de ceux qui, à l'instar de l'historien Michel Bock, n'auraient pas bien saisi le caractère provincialiste de la pensée de Groulx (p. 13-14).

Ainsi campé, l'ouvrage propose une radioscopie des groupes indépendantistes qui, formés d'intellectuels plutôt jeunes, tentent de faire la promotion de l'indépendance, ce que l'auteur voit comme la formation d'un réseau indépendantiste. En dépouillant leur correspondance, il montre à quel point, dans ces efforts, Groulx agissait comme le principal mentor ou guide auprès de qui ces jeunes cherchaient une caution intellectuelle. D'autres penseurs agissaient aussi comme référence intellectuelle, Édouard Montpetit, par exemple, qui inspirait les membres du mouvement Jeune-Canada en matière économique. Mais, ainsi que Noël l'écrit, l'abbé Groulx était bien l'inspiration de ces premiers intellectuels indépendantistes - leur chef spirituel -, et celui qui « manœuvr[ait] à l'arrière-scène " (p. 67). 
Les chapitres deux et trois sont particulièrement intéressants puisque l'auteur nous invite, si on peut le dire ainsi, à visiter l'intérieur du réseau. D'abord, il s'intéresse au groupe Jeune-Canada qui a pris son envol en 1932, tout en demeurant un groupe modeste qui ne dépassera pas 20 membres. Il montre que le groupe, bien que non partisan, espérait la défaite des libéraux et appuyait la coalition Gouin-Duplessis. Fortement soudés, ces intellectuels, André Laurendeau étant le plus influent, offraient une critique forte de la société québécoise, laquelle s’inscrivait dans l'esprit de celle de Groulx à qui ils étaient restés " fidèles ». Comme Groulx, ils voulaient que la jeunesse canadienne-française s'engage davantage, d'où la nécessité d'une éducation résolument nationale (p. 46-47). Noël montre qu'ils étaient fortement tentés par l'aventure indépendantiste, comme l'illustre une lettre d'André Laurendeau adressée à Groulx, en août 1933, dans laquelle il se demandait s'il n’était pas nécessaire «d'aider le destin ». Rejetant la thèse des réformes constitutionnelles, craignant la montée du marxisme dans certains coins du pays, les membres de Jeune-Canada embrassaient l'idée d'un État francophone occupant la Laurentie, un territoire aux frontières indéterminées. Évidemment, le tout restait fort imprécis, mais les francophones hors Québec étaient, semble-t-il, abandonnés à leur sort. Par ailleurs, certains membres du groupe ne se laisseront pas convaincre par l'idée d'indépendance, Pierre Dansereau notamment, qui préféra quitter le groupe (p. 55).

Par la suite, le groupe de Québec, qui fonde le journal La Nation en février 1936, éclipse en quelque sorte le mouvement Jeune-Canada, qui entrera dans une phase de retrait. La fondation de cette publication a marqué, nous dit l'auteur, un tournant pour le réseau puisque l'indépendantisme canadien-français s'imposait comme étant «l'objectif déclaré » du journal (p. 66). En ce sens, un pas supplémentaire avait été franchi. À Montréal, ce sont les Jeunesses patriotes qui se sont lancées dans la mêlée avec, à leur tête, Walter O'Leary. L'époque était à l'effervescence et le ton se radicalisait. Cherchant en effet un modèle du côté de l'Italie mussolinienne, Paul Bouchard ne demandait 
rien de moins, dans La Nation du 15 février 1936, que l'abolition de la démocratie et son remplacement par le fascisme (p. 86). Il attendait un chef fort qui défendrait les préceptes du modèle corporatiste. Mais le corporatisme est demeuré davantage un mot d'ordre, car on ne trouvait pas, du moins Noël n'en parle pas, d'équivalent institutionnel de cette idée. En outre, dans ce discours de plus en plus radical, les immigrants étaient mal vus, spécialement les juifs. Enfin, comme pour les membres de Jeune-Canada, les indépendantistes de Vivre et de La Nation vouaient une grande admiration à Groulx même si, précise Noël, ce dernier n'occupait plus la place prépondérante qui était la sienne aux premières années du mouvement.

À partir de 1937, le mouvement indépendantiste va s'essouffler sous le poids des querelles et des rivalités entre des individus qui se connaissaient bien, probablement aussi en raison de la déception causée par le gouvernement de Maurice Duplessis, qui a écarté les éléments les plus radicaux, sans compter la menace de la conscription qui hantait alors les esprits et qui se concrétisera d'ailleurs par la suite (p. 105). Quelques apôtres esseulés continueront le combat comme Dostaler O'Leary ou encore Wilfrid Morin, qui ne faisait pas partie du réseau des années 1930, mais qui, avec Nos droits à l'indépendance politique (1938), en poursuivit l'idée.

Ce regard de l'intérieur permet de restituer les débats soulevés par l'émergence d'une pensée plus revendicatrice sur le plan politique, dans le contexte de la montée des fascismes européens. Certes, Noël ne tranche pas vraiment la question des convictions indépendantistes de Groulx lui-même puisqu'il n’offre pas, en définitive, de réponse catégorique. En revanche, il montre que Groulx a certainement été celui qui a exercé le plus d'influence auprès des jeunes intellectuels de l'époque, qu'il a connu la tentation de l'indépendantisme et qu'il a favorisé l'effort de la nouvelle garde intellectuelle, mais sans jamais se résoudre à l'embrasser résolument. Ainsi, Groulx ne peut être écarté de la genèse de l'indépendantisme, et c'est pourquoi ce travail possède une réelle valeur pour qui veut comprendre les premiers balbutiements de cette idée. À l'instar de certains autres travaux, cet ouvrage rappelle 
qu'on ne peut parler de rupture complète entre le nationalisme canadien-français et celui qui émergera au moment de la Révolution tranquille. Il s'agit donc d'une pièce de plus à ajouter au dossier des études de la pensée groulxiste ainsi qu'à celui de l'histoire de l'idée nationale au Québec.

\section{- Frédéric Boily \\ Campus Saint-Jean \\ Université de l'Alberta}

\section{Alan Gordon. The Hero and the Historians: Historiography and the Uses of Jacques Cartier, Vancouver, UBC Press, 2010, 248 p.}

Auteur d'un livre important sur le processus de commémoration dans la ville de Montréal de 1891 à 1930, Alan Gordon nous offre maintenant une analyse de l'héritage historiographique de Jacques Cartier. Dans son ouvrage, il analyse les récits des voyages de Jacques Cartier à partir $\mathrm{du} \mathrm{XvI}^{\mathrm{e}}$ siècle, qui ont généralement été écrits par des historiens œuvrant à l'extérieur des institutions universitaires. L'explorateur réalise trois voyages plus ou moins troublés en Amérique du Nord dans les années 1530-1540. Les relations qu'il a écrites représentent la principale source utilisée dans la rédaction des récits de ses voyages.

Comme plusieurs autres personnages historiques considérés comme des héros dans l'histoire du Québec et du Canada, Cartier atteint le sommet de sa renommée à la fin du XIX ${ }^{\mathrm{e}}$ siècle et au début du XXe siècle. Presque oublié au XVII ${ }^{\mathrm{e}}$, au XVIII ${ }^{\mathrm{e}}$ et au début du XIX siècle, Cartier occupe une place de premier plan à l'approche du $300^{\mathrm{e}}$ anniversaire de son premier voyage. Dès ce moment, il inspire en particulier des nationalistes canadiens-français, qui voient en lui le père de la Nouvelle-France. Il représente pour eux un héros catholique et royaliste. Comparé surtout à Samuel de Champlain, Cartier lui dispute le titre de fondateur du pays. Contrairement à certains autres personnages historiques, il est difficile d'imaginer une perspective 